\title{
Home visiting to an HIV positive patient prior to initiation of HAART has Lasting impact on their adherence. TASO Experience
}

\author{
Emmanuel Odeke*, Ricky Jones Nyatia, Peter Sekiranda \\ From $16^{\text {th }}$ International Symposium on HIV and Emerging Infectious Diseases \\ Marseille, France. 24-26 March 2010
}

\section{Background}

Adherence to life saving Anti-retroviral drugs (ARVs) is important to the success of antiretroviral therapy (ART) programs. In order to achieve optimum viral suppression an adherence level of $>95 \%$ is required.

Readiness of the patient to take medication for life during the initiation process of ART is one of the key criteria used. If this criterion is under looked it has got adverse consequences on maintaining high level of adherence.

The AIDS Support organization (TASO) put emphasis on patients' readiness and presence of family support as one of the criteria for ART initiation.

\section{Methods}

The AIDS Support Organization (TASO) is a national non governmental organization in Uganda, whose mission is preventing HIV infection, restoring hope and improving the quality of life of individuals, families and communities infected and affected by HIV and disease.

Patients are usually screened for ART eligibility by CD4 and WHO staging. When the patient is found to be eligible a home visit is arranged for with the patient consent to. During the home visit, which is done by a counselor/field staff, psychological and social preparation is done. This involves seeking for long lasting family support to the patients, identification and addressing barriers to adherence in the home, and identification of a medicine supporter. Also during the home visit adherence plan is made by the patient and this is routi- nely reviewed during the subsequent counseling sessions.

\section{Results}

Retrospective analysis of the data at one of the centers (TASO Masindi). 78\% of patients on ART were assessed for readiness for ART through home visit prior to initiation of ART. Out of these $85 \%$ had adherence level $>95 \%$ while $78 \%$ of the patients not home visited had adherence level of $<95 \%$.

Other findings were that, the proportion of patients on ART disclosing sero-status to family members has increased. Follow-up of Patient's in case of non adherence has been made easy since it is easy to review the adherence plans that have been made.

\section{Discussion}

Addressing psychological and social factors during a home visit contributes significantly to good adherence for people living with HIV/AIDS.

Published: 11 May 2010

doi:10.1186/1742-4690-7-S1-P155

Cite this article as: Odeke et al.: Home visiting to an HIV positive patient prior to initiation of HAART has Lasting impact on their adherence. TASO Experience. Retrovirology 2010 7(Suppl 1):P155.

\footnotetext{
* Correspondence: emmanuel_odeke@yahoo.com

The AIDS Support Organisation (TASO), Kampala, Uganda
} 